\title{
Form-finding of shells containing both tension and compression using the Airy stress function
}

\author{
Masaaki Miki ${ }^{1}$, Emil Adiels ${ }^{2}$, William Baker ${ }^{3}$, Toby Mitchell $^{3}$, Alexander Sehlström² and Chris \\ J. K. Williams ${ }^{2}$
}

\begin{abstract}
Pure-compression shells have been the central topic in the form-finding of shells. This paper studies tensioncompression mixed type shells by utilizing a NURBS-based isogeometric form-finding approach that analyzes Airy stress functions to expand the possible plan geometry. A complete set of smooth version graphic statics tools is provided to support the analyses. The method is validated using examples with known solutions, and a further example demonstrates the possible forms of shells that the proposed method permits. Additionally, a guideline to configure a proper set of boundary conditions is presented through the lens of asymptotic lines of the stress functions.
\end{abstract}

\section{Keywords}

force diagram, Airy stress function, graphic statics, form-finding, membrane shell, tension-compression mixed type shell, asymptotic lines, wave equation

\section{Introduction}

Shell structures, such as concrete, masonry, and metal shells, steel or timber gridshells, cable nets, and fabric structures, are elegant and light-weight (Fig. 1). Their dominating load-carrying action is membrane action, which is the combination of tensile, compressive, and shear stresses or forces acting in a plane tangential to the surface of the structure. Shell structures containing only tensile or only compressive stresses can not have unsupported boundaries with overhang but must have such that lean 'inward' (Fig. 2).

To allow for overhangs, a mix of tension and compression stresses are needed (Fig. 3). Whenever there are compressive stresses, some bending stiffness is required to avoid buckling. Bending stiffness can also be added to allow for the shape of the shell to deviate from the pure membrane action shape (Fig. 4).

During design of shell structures, membrane action is commonly secured using structural form-finding whereby a geometry is determined in such a way that no bending action is needed for the load transfer of the dominating load. Most numerical methods for form-finding of shells simulate a physical model that might involve hanging chains or fabric that inverted form a compression structure. The models are usually discretized using line elements connected to mutual nodes where external loading also is applied, and the geometry is iteratively updated based on the previously computed stress state. As a result, such methods are restricted to tensile only or compressive only shell structures.

In this paper, we revisit, clarify, and develop the numerical form-finding method presented by Miki et al. ${ }^{1}$ and the smoooth version Graphic Statics tools discussed discussed in Miki et al. ${ }^{2}$. The method takes as input a pre-determined membrane stress state specified using the scalar-valued Airy stress function ${ }^{3} \phi$ defined on a plane. With $\phi$ given, the shape of the shell $z$ is solved such that the vertical equilibrium

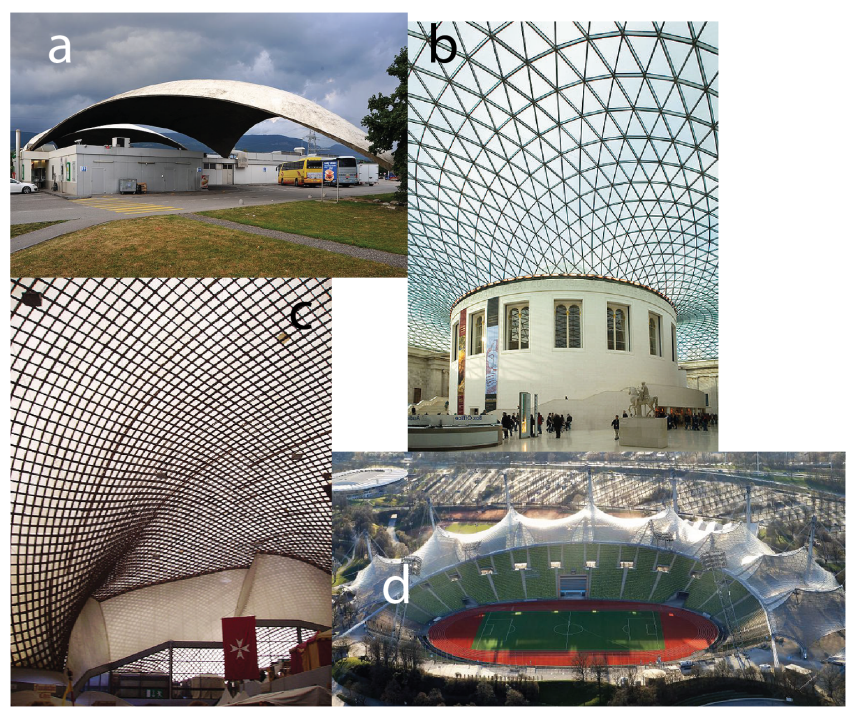

Figure 1. Compression shells and lightweight tensile structures: (a) Highway service area Deitingen South by Heinz Isler, (C)Хрюша, 2009; (b) British Museum Great Court Roof by Fosters and Partners in collaboration with Chris Williams, (C)Andrew Dunn, 2005; (c) Multihall in Mannheim by Frei Otto, (C)Immanuel Giel, 2006; and (d) Olympic Stadium in Munich by Frei Otto, (C) Meister Eiskalt, 2014

\footnotetext{
${ }^{1}$ The University of Tokyo, Japan

${ }^{2}$ Department of Architecture and Civil Engineering, Chalmers University of Technology, Sweden

${ }^{3}$ Skidmore, Owings and Merrill, USA
}

\section{Corresponding author:}

Masaaki Miki, The University of Tokyo, Komaba3-8-1, Meguro, Tokyo, 153-0041.

Email: masaakim@g.ecc.u-tokyo.ac.jp 


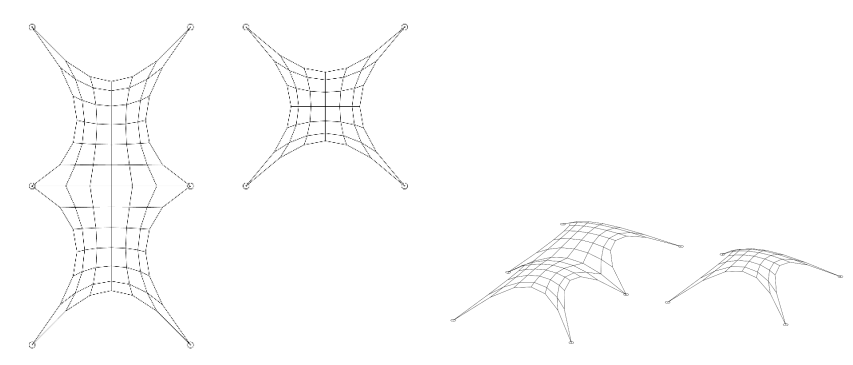

Figure 2. Plans (left) and bird's-eye view (right) of pure compression gridshells that have open edges on the boundary.

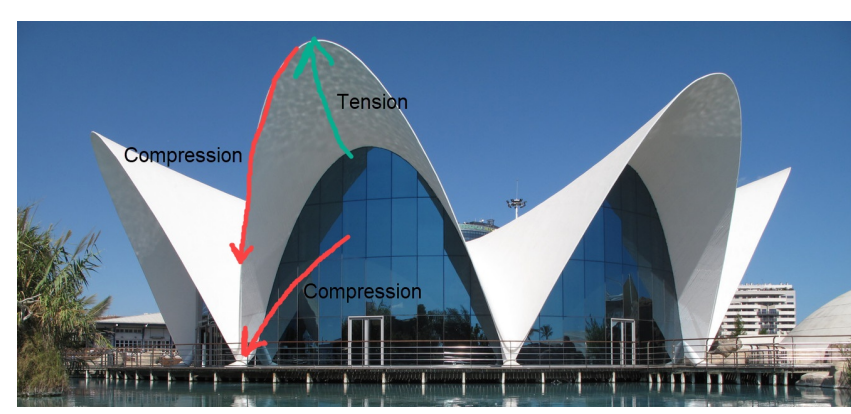

Figure 3. Félix Candela's hyperbolic-paraboloidal reinforced concrete shell for the Los Manantiales restaurant at Xochimilco.

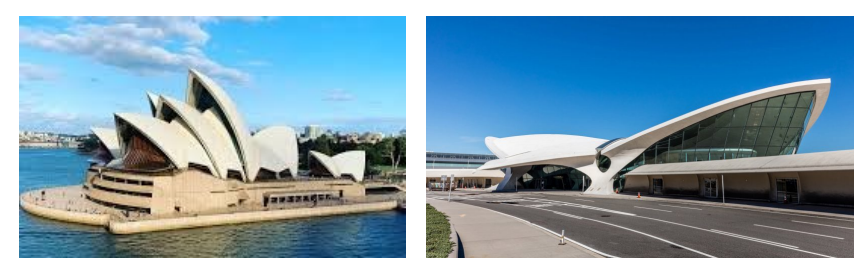

Figure 4. Sydney Opera House by Jorn Utzon (left) and JKF Terminal by Eero Saarinen (right).

equation

$$
\frac{\partial^{2} \phi}{\partial x^{2}} \frac{\partial^{2} z}{\partial y^{2}}-2 \frac{\partial^{2} \phi}{\partial x \partial y} \frac{\partial^{2} z}{\partial x \partial y}+\frac{\partial^{2} \phi}{\partial y^{2}} \frac{\partial^{2} z}{\partial x^{2}}=-\rho,
$$

is satisfied ${ }^{4,5}$ given an appropriate set of boundary conditions. $\rho$ is the upward vertical loading per unit plan area, which may be constant or dependent on the shape. Eq. (1) is equivalent to Eq. (f) on page 462 in Theory of Plates and Shells ${ }^{6}$, whose authors attribute the first use of the Airy stress function in this manner to Pucher ${ }^{7}$. Csonka discusses in chapter 11 and the subsequent chapters of Theory and Practice of Membrane Shells stress functions for shells on polygonal plans in pure compression and starshaped plans with a mix of compression and tension ${ }^{5}$.

Some shell structures consist of several smooth surfaces joined via sharp kinks where concentrated forces arise, for example, several of Félix Candela's concrete shells (Fig. 3) and Jorn Utzon's Sydney Opera house (Fig. 4, left). Such force concentrations can be represented in the Airy stress function by narrow regions with a high degree of curvature. If these narrow regions are taken to the limit, 'folds' forms on the stress function ${ }^{8}$. Then the piecewise smooth stress function can be seen as a hybrid between a smooth stress function and a stress polyhedral ${ }^{9}$.

In graphic statics for bar frameworks such as planar trusses, stress polyhedrons can be used to establish a discrete force diagram ${ }^{10-14}$ which has a reciprocal relation to a discrete form diagram. Graphic statics originate in the 18th and 19th century ${ }^{15-20}$ and has recently regained attention ${ }^{21-30}$ with generalization into higher dimensions ${ }^{31-38}$ and applications for the form-finding of shells ${ }^{14,39-44}$. Attempts have been made to establish a similar relation between a piecewise smooth stress function and the shape of the shell which require the computation of the Christoffel symbols of the second kind ${ }^{1}$. To avoid the often tedious computation of the Christoffel symbol, a reciprocal relation between the piecewise smooth continuous stress function, a pre-computed continuous force diagram, and continuous form diagram (i.e. plan geometry), has been established $^{2}$.

If $\phi$ has positive Gaussian curvature, it represents a state of pure compression or pure tension, whereas if the Gaussian curvature is negative, it represents a state of compression and tension. Thereby the method, in principle, allows for the form-finding of shells with any membrane stress state. However, the stress functions considered in this paper all have negative Gaussian curvature resulting in mixed tension-compression shell structures. For such cases, Eq. (1) becomes a hyperbolic partial differential equation ${ }^{4}$ often challenging to solve analytically.

In line with the methodology by Miki et al. ${ }^{1}$, Eq. (1) is solved numerically using isogeometric analysis with NURBS surfaces as finite elements ${ }^{45-48}$. With isogeometric analysis, the computational model and the geometry model is the same, eliminating the need to discretize smooth shell surface into flat panels or a network of straight-line elements. Without the need to mesh the surface, designers can concentrate on the the form in early design stages, leaving descritization of the surface into structural elements such as panels and bars for later stages.

\subsection{Contributions}

In this paper, we breakdown the original method presented by Miki et al. ${ }^{1}$ to make it more accessible and reproducible. While the earlier paper ${ }^{1}$ restricted its attention to shells of pure compression, we concentrate on shells containing both compression and tension stresses. The increments in this paper are as follows:

1. Provides a complete set of smooth version graphic statics tools through stress function, form diagram, and force diagram (Section 2).

2. Revisits the numerical form-finding method discussed in Miki et al. ${ }^{1}$ (Section 2). Minor revisions include:

(a) The stress function and the shell can have different parametric representations.

(b) Linear springs are provided to constrain any point to a desired height.

3. Known solutions of tension-compression mixed type shells are used to validate the method (Section 3).

4. A guideline to choose proper boundary conditions for tension-compression mixed type problem is addressed (Section 4) through the lens of asymptotic lines.

5. Introduces an Airy stress function (Section 5) that can take an arbitrary plan geometry. An example problem is provided to demonstrate the possible forms enabled by the proposed stress functions. 


\section{Theoretical background and numerical method}

In this section, starting from the traditional discrete graphic statics tools, their smooth versions are derived. Then, a NURBS-based isogeometric form-finding method is introduced. The method solves a linear system of equations when the surface area is evaluated on the projected plane. Its nonlinear version that accounts for the surface area accurately is also presented.

\subsection{Discrete force diagrams and Airy stress polyhedron}

Figure 5 shows an example of a force and form diagram pair. If each internal node in the form diagram is balanced by the axial forces acting along the incoming lines, a reciprocal force diagram can be constructed consisting of closed polygons. If the form diagram is not in equilibrium, one or several of the polygons in the force diagram can not be closed. In the force diagram, the edges of the polygons have lengths representing the magnitude of the corresponding force and are rotated by 90 compared to the corresponding line in the form diagram ${ }^{49}$. The reciprocal relation is such that a point, a polygon, and a line in a form diagram maps to a polygon, a point, and a line in a force diagram, respectively.

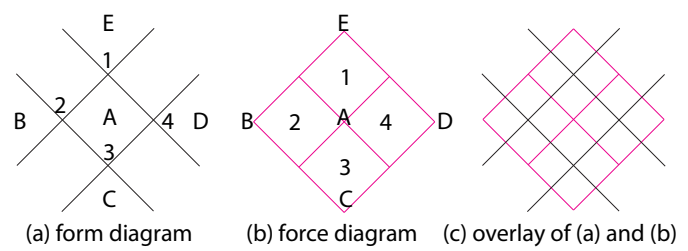

Figure 5. An example of form and force diagrams.

The existence of a force diagram is equivalent to the existence of planar faced polyhedron (i.e. stress polyhedron $^{9}$, or Airy stress polyhedron) whose planar projection is the form diagram ${ }^{10-14}$. If the plane of a face in such a polyhedron is expressed as

$$
a x+b y+c=\phi,
$$

its corresponding point in the force diagram is located at $(a, b)$. At the same time, the 'normalized' normal vector of the same face is given by

$$
\boldsymbol{N}=(a, b,-1)
$$

Thus, a force diagram can be obtained by flattening all the normal vectors of the polyhedron and connecting the points if their corresponding faces are adjacent (Fig. 6).

\subsection{Continuous force diagram}

For a stress function $\phi=\phi(x, y)$, the normalized normal vector is given by

$$
\boldsymbol{N}=\left(\frac{\partial \phi}{\partial x}, \frac{\partial \phi}{\partial y},-1\right)
$$

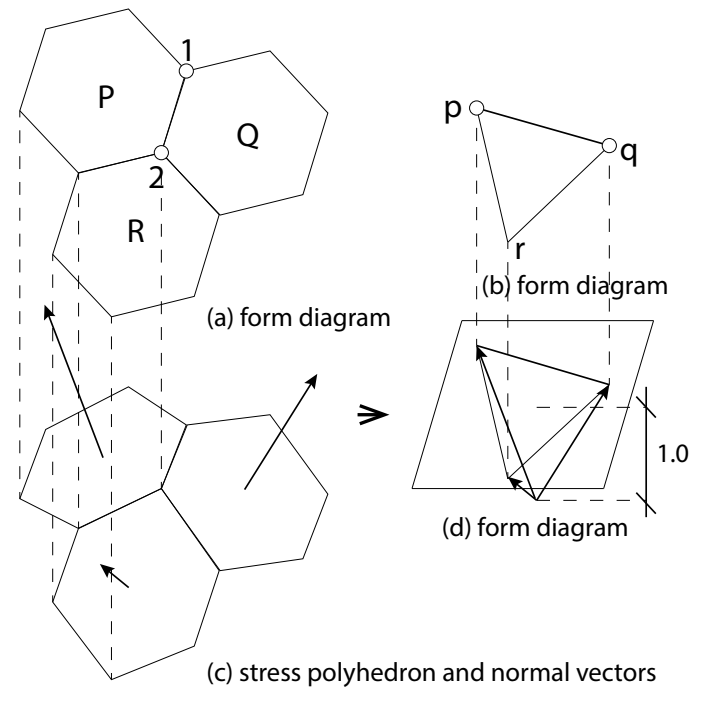

Figure 6. Stress polyhedron and face normal vectors.

and the second Piola-Kirchhoff (PK) stress tensor ${ }^{50}$ for the projected geometry of the shell can be computed as

$$
S=\left[\begin{array}{ll}
S_{x x} & S_{x y} \\
S_{y x} & S_{y y}
\end{array}\right]=\left[\begin{array}{cc}
\frac{\partial^{2} \phi}{\partial y^{2}} & -\frac{\partial^{2} \phi}{\partial y \partial x} \\
-\frac{\partial^{2} \phi}{\partial x \partial y} & \frac{\partial^{2} \phi}{\partial x^{2}}
\end{array}\right]
$$

Since the order of differentiation of $\phi$ does not matter,

$$
\frac{\partial}{\partial y \partial x}=\frac{\partial}{\partial x \partial y}
$$

any choice of $\phi$ automatically satisfies the horizontal equilibrium equations

$$
\left.\begin{array}{c}
\frac{\partial S_{x x}}{\partial x}+\frac{\partial S_{x y}}{\partial y}=\frac{\partial}{\partial x} \frac{\partial^{2} \phi}{\partial y^{2}}-\frac{\partial}{\partial y} \frac{\partial^{2} \phi}{\partial x \partial y}=0 \\
\frac{\partial S_{x y}}{\partial x}+\frac{\partial S_{y y}}{\partial y}=\frac{\partial}{\partial y} \frac{\partial^{2} \phi}{\partial x^{2}}-\frac{\partial}{\partial x} \frac{\partial^{2} \phi}{\partial x \partial y}=0
\end{array}\right\} .
$$

So dose any pair of functions $X$ and $Y$ that satisfy

$$
\frac{\partial Y}{\partial x}=\frac{\partial X}{\partial y} \Leftrightarrow \frac{\partial Y}{\partial x}-\frac{\partial X}{\partial y}=0 .
$$

For such a pair, the stress tensor is given by

$$
S=\left[\begin{array}{ll}
S_{x x} & S_{x y} \\
S_{y x} & S_{y y}
\end{array}\right]=\left[\begin{array}{cc}
\frac{\partial Y}{\partial y} & \frac{-\partial X}{\partial y} \\
-\frac{\partial Y}{\partial x} & \frac{\partial X}{\partial x}
\end{array}\right]
$$

Thus, from Eqs. (5) and (9),

$$
X=\frac{\partial \phi}{\partial x}, \quad Y=\frac{\partial \phi}{\partial y},
$$

which are the components of the normal vector in Eq. (4). Hence, in analogy with the relationship between the normal vector of a stress polyhedron and the discrete force diagram, $(X, Y)$ is the continuous force diagram corresponding to a continuous stress function ${ }^{2}$.

Note that the existence of a stress function $\phi$ is not necessary to construct a force diagram $(X, Y)$. In fact, 
it is sufficient to require the conditions given in Eq. (8). This condition is known as 'curl-free,' and is essentially an integrability condition of gradient vectors. It is also explained in a structured manner in exterior calculus ${ }^{51}$.

Curl-free means that the deformation gradient

$$
F=\left[\begin{array}{ll}
F_{x x} & F_{x y} \\
F_{y x} & F_{y y}
\end{array}\right]=\left[\begin{array}{ll}
\frac{\partial X}{\partial x} & \frac{\partial X}{\partial y} \\
\frac{\partial Y}{\partial x} & \frac{\partial Y}{\partial y}
\end{array}\right]
$$

is symmetric and consequently has no components of rotation. Force diagram can be considered as a deformed shape of the form diagram, and the local deformation at each point can be described by a deformation gradient. The deformation gradient maps a unit circle in the form diagram (Fig. 7, a) to an ellipsoid in the force diagram (Fig. 7, b). Without any rotational deformations, there are two orthogonal directions where the local deformation has stretches only (the red lines in the figure) and these coincide with the principal directions of the stress tensor but the first and the second directions are swapped (Fig.7, b).

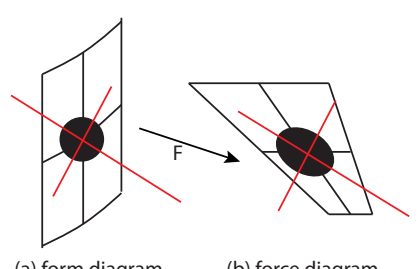

(a) form diagram

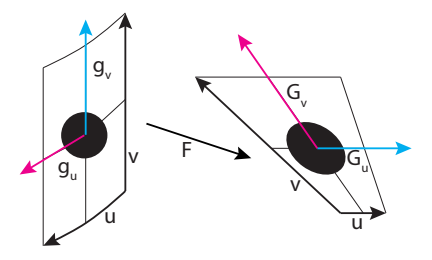

(a) form diagram

(b) force diagram

Figure 8. Perpendicular base vectors. deformation.

\subsection{Introduction of curvilinear coordinate system}

So far, an orthonormal coordinate system $(x, y)$ has been used. However, for shells with arbitrary boundary shape, a curvilinear coordinate system $(u, v)$ is often preferable. The mapping between the two systems is a matter of differential geometry ${ }^{52}$ and books such as Ciarlet ${ }^{53-55}$ discuss this from a theory of elasticity point of view.

Let $(u, v) \rightarrow(x, y)$ and $(u, v) \rightarrow(X, Y)$ be two symmetrical maps. Then the maps $(u, v) \rightarrow(x, y)$ and $(u, v) \rightarrow$ $(X, Y)$ define a pair of parametric surfaces representing the form and force diagrams that can be expressed as

$$
\boldsymbol{x}(u, v)=(x(u, v), y(u, v))
$$

respectively

$$
\boldsymbol{X}(u, v)=(X(u, v), Y(u, v)) .
$$

Because the inverse of $(u, v) \rightarrow(x, y)$ exists, $(x, y) \rightarrow$ $(X, Y)$ also exits. If it is given by Eq. (10), it can be computed by

$$
\begin{aligned}
& X=\frac{\partial \phi}{\partial x}=\frac{\partial \phi}{\partial u} \frac{\partial u}{\partial x}+\frac{\partial \phi}{\partial v} \frac{\partial v}{\partial x} \\
& Y=\frac{\partial \phi}{\partial y}=\frac{\partial \phi}{\partial u} \frac{\partial u}{\partial y}+\frac{\partial \phi}{\partial v} \frac{\partial v}{\partial y}
\end{aligned}
$$

where

$$
\left[\begin{array}{ll}
\frac{\partial u}{\partial x} & \frac{\partial u}{\partial y} \\
\frac{\partial v}{\partial x} & \frac{\partial v}{\partial y}
\end{array}\right]=\left[\begin{array}{ll}
\frac{\partial x}{\partial u} & \frac{\partial x}{\partial v} \\
\frac{\partial y}{\partial u} & \frac{\partial y}{\partial v}
\end{array}\right]^{-1}
$$

The deformation tensor $F$ on a $(u, v)$ system is known ${ }^{56}$ as

$$
F=\boldsymbol{G}_{u} \otimes \boldsymbol{g}^{u}+\boldsymbol{G}_{v} \otimes \boldsymbol{g}^{v},
$$

where $\boldsymbol{g}^{u}$ and $\boldsymbol{g}^{v}$ are the base vectors of $\boldsymbol{x}(u, v)$ and $\boldsymbol{G}_{u}$ and $\boldsymbol{G}_{v}$ are the base vectors of $\boldsymbol{X}(u, v)$. For details on the computation of the base vectors and the extraction of the deformation components $F_{x x}, F_{x y}=F_{y x}$, and $F_{y y}$, see the Appendix A.

The deformation gradient tensor $F$ given by Eq. (16) is equivalent to the one given by Eq. (11) and both are symmetric. Therefore,

$$
\boldsymbol{G}_{u} \cdot \boldsymbol{g}_{v}=\boldsymbol{G}_{v} \cdot \boldsymbol{g}_{v}
$$

which is an equivalent condition of curl-free. A special case of curl-free is when $\boldsymbol{G}_{u} \cdot \boldsymbol{g}_{v}=\boldsymbol{G}_{v} \cdot \boldsymbol{g}_{u}=0$ (Fig. 8), which is an interpretation of the perpendicular condition of the discrete force diagrams to the continuous ones.

In general, a computation of the second derivatives of the stress function on a curvilinear coordinate system requires the computation of the Christoffel symbols of the second kind, $\Gamma_{i j}^{k}$, describing the curvature of the isocurves of a parametric surface (see e.g. Green and Zerna ${ }^{4}$, Eisenhart ${ }^{52}$, Ciarlet ${ }^{53-55}$ ). However, through the above formulation, the first differentiation is computed on $(u, v)$, yielding a vector on $(u, v)$ system that is transformed to the $(x, y)$ system using Eq. (14). Then, a differentiation of $(X, Y)$ with respect to $(u, v)$ is computed using Eq. (16), but $(X, Y)$ is already a vector on $(x, y)$ system. Therefore, there is no need to compute the Christoffel symbols. Moreover, the precomputed force diagram, $(X, Y)$, assists the evaluation of the crease curves in a piecewise smooth stress function (Section 2.7). This is where the proposed method takes advantage of the original method by Miki et al. ${ }^{1}$, substantially simplifying the computations.

\subsection{Linear method}

The horizontal equilibrium of the shell is ensured by Eq. (7). To complete the equilibrium, the shape of the shell $z$ is to be determined so that the vertical equilibrium given by Eq. (1) is fulfilled. Using the principle of virtual work, a finite number of simultaneous equations can be obtained as an approximation of the vertical equilibrium equation. This approach is equivalent to the Galerkin method ${ }^{57}$ and the details are laid out in the Appendix B.

Assume the vertical loading $\rho$ constant so that the solution $z$ of Eq. (1) becomes a linear problem. Later on, in Section 2.5, $\rho$ will be taken as the self-weight of the shell. Then $\rho$ is no longer constant but dependent on the actual area of the shell, turning Eq. (1) into a non-linear problem.

Introduce a curvilinear coordinate system $(\xi, \eta)$ on the shell that is different from that of the force and force diagrams $(u, v)$. Let $\boldsymbol{h}_{\xi}$ and $\boldsymbol{h}_{\eta}$ be the base vectors and $h_{i j}$ be the first fundamental form of the $(\xi, \eta)$ system, and denote the reference states of the properties of the shell that are not 
affected by differential operators by a bar ${ }^{-}$. Then from the principle of virtual work follows

$$
\delta w=\sum_{\text {surfaces }} \int\left(\frac{1}{2} \bar{S}^{i j} \delta h_{i j}-\bar{\rho} \delta z\right) \mathrm{d} \bar{a}=0,
$$

where

$$
\mathrm{d} \bar{a}=\sqrt{\operatorname{det} \bar{h}_{i j}} \mathrm{~d} \xi \mathrm{d} \eta
$$

is a small area element, $\bar{S}^{i j}$ the components of the 2nd PK stress tensor re-evaluated on $(\xi, \eta)$, and $\bar{\rho}$ a constant loading per unit area, all measured in the plan of the form diagram. Einstein summation convention applies and the indices $i$ and $j$ are either 1 or 2 , where 1 corresponds to the $\xi$ direction and 2 the $\eta$-direction. Details on the extraction of the stress components on the $(\xi, \eta)$ system are given in the Appendix A.

If the shell is represented by multiple finite elements or parametric surfaces (e.g. triangles, quadrilaterials, or NURBS-surfaces) defined by $\mathrm{n}$ independent control points, all functions defined on the shell, such as $h_{i j}$ and $z$, become functions of the control point coordinates. By packing the coordinates of these control points in a 3n-dimensional column vector $\boldsymbol{r}$ and replacing the variation of functions $\delta f$ by $\nabla f \delta \boldsymbol{r}$, where $\delta \boldsymbol{r}$ is a 3 n-dimensional arbitrary column vector and the gradient operator is

$$
\nabla=\left[\frac{\partial}{\partial r_{1}}, \cdots, \frac{\partial}{\partial r_{3 \mathrm{n}}}\right]
$$

Eq. (18) becomes

$$
\sum_{\text {surfaces }} \int\left(\frac{1}{2} \bar{S}^{i j} \nabla h_{i j}-\bar{\rho} \nabla z\right) \mathrm{d} \bar{a}=\mathbf{0} .
$$

Since the horizontal equilibrium is automatically ensured, solving Eq. (21) in terms of $z$ is enough to obtain a complete equilibrium. In case Eq. (21) is solved in terms of $x$ and $y$, the plan of the solution should match the form diagram.

Note that Eq. (21) is linear in $\boldsymbol{r}$. By distributing integration points and performing a numerical integration (see Section 2.8), a system of linear equations is obtained. With a sufficient number of fixed points given, this system of equations can be solved easily by computing an inverse matrix. Although presented quite differently, Pauletti and Pimenta ${ }^{58}$ have already pointed out that having a constant second PK stress tensor results in a linear system of equations.

\subsection{Nonlinear iterative method}

If $\bar{\rho}$ is the self-weight of the shell, Eq. (18) only gives an approximate solution. The exact solution is given by

$$
\delta w=\sum_{\text {surfaces }} \int\left(\frac{1}{2} \bar{S}^{i j} \delta h_{i j} \mathrm{~d} \bar{a}-\bar{\rho} \delta z \mathrm{~d} a\right)=0,
$$

where

$$
\mathrm{d} a=\sqrt{\operatorname{det} h_{i j}} \mathrm{~d} \xi \mathrm{d} \eta
$$

is the actual element area measured on the shell. Since $\mathrm{d} a$ is dependent on $h_{i j}$ that is a property of the sought solution $z$, Eq. (22) is a non-linear problem requiring an iterative solution process.
In each iterative step, the residual forces of the system are computed by

$$
\boldsymbol{R}=\sum_{\text {surfaces }} \int \boldsymbol{r}^{T}\left(\frac{1}{2} \bar{S}^{i j} \nabla^{2} h_{i j} \mathrm{~d} \bar{a}-\bar{\rho} \nabla z \mathrm{~d} a\right),
$$

where $\nabla^{2}=\nabla^{T} \nabla$ and $\nabla^{2} h_{i j}$ is a constant matrix. Thus, the matrix $\bar{S}^{i j} \nabla^{2} h_{i j}$ does not change during the iterations.

Then, with $h^{i j}$ the inverse of $h_{i j}$ and using the relation

$$
\mathrm{D} \sqrt{\operatorname{det} h_{i j}}=\frac{1}{2} h^{i j} \mathrm{D} h_{i j} \sqrt{\operatorname{det} h_{i j}}
$$

where $\mathrm{D}$ is a generic differential operator that can be either a $\delta, \mathrm{d}$, or $\nabla$ operator, the system of equations to solve in each step becomes

$$
\begin{array}{r}
\frac{1}{2}\left[\sum_{\text {surfaces }} \int\left(\bar{S}^{i j} \nabla^{2} h_{i j} \mathrm{~d} \bar{a}-\bar{\rho} h^{i j} \nabla z^{T} \nabla h_{i j} \mathrm{~d} a\right)\right] \Delta \boldsymbol{r} \\
=-\boldsymbol{R}
\end{array}
$$

Note that in the first step, solving Eq. (26) is the same as solving Eq. (21). The convergence of the solution is often improved when the second term in the parentheses of Eq. (26) is omitted, i.e. it is equivalent to repeating the linear method but with the updated loading, and $\delta \boldsymbol{r}$ is scaled down by 0.5 .

\subsection{Point and area supports}

Fixing a control point to a specific height is easily done by eliminating the parameter completely from the system of equations. However, if NURBS surfaces are used as finite elements, the control points do not pass through the surface except at the corner points. So, to constrain an arbitrary point of such a surface to a specific height, a linear spring can be used.

The elastic energy of such a spring is

$$
\frac{1}{2} k(z(\boldsymbol{r})-\bar{z})^{2}
$$

where $\bar{z}$ is the prescribed height of the point, $z(\boldsymbol{r})$ is a linear function that converts the control points to the height of the point, and $k$ is a penalty factor.

When such springs are incorporated into the system, the equilibrium of the entire system is

$$
\sum_{\text {springs }} k\left(\boldsymbol{r}^{T} \nabla z^{T} \nabla z-2 \bar{z} \nabla z\right)+\text { membrane term }=\mathbf{0},
$$

where the spring term is linear in $r$ and the membrane term is discussed in Section 2.4 or 2.5 depending on loading condition.

Similarly, area supports defined by closed curves can be imposed on the shell by the use of a set of linear springs distributed along the curve. This approach is used later on in Section 5 when discussing some examples.

\subsection{Piecewise smooth stress functions}

Whenever the shell is made up of several smooth surfaces joined by sharp kinks along their intersection curves, the stress function must be a piecewise smooth function. For 
such shells, the equilibrium is sustained by concentrated forces acting along the kinks on the shell. The axial force along a kink in the shell surface can be calculated by measuring the 'jump' of the normal vectors on either side of the corresponding kink in the stress function. The same entity can be found by measuring the 'jump' in the force diagram. Denoting the 'jump' by $\bar{L}$, the 2 nd PK stress tensor along a kink in the surface ${ }^{1}$ is

$$
\bar{n}^{11}=\left(\bar{h}_{11}\right)^{-1} \bar{L}
$$

and the principle of virtual work adds up to Eq. (18) or Eq. (22) is

$$
\delta w=\sum_{\text {curves }}\left(\int \frac{1}{2} \bar{n}^{11} \delta h_{11} \mathrm{~d} \bar{\ell}\right)
$$

where

$$
d \bar{\ell}=\sqrt{\operatorname{det} \bar{h}_{11}}
$$

is a small length element on the intersection curve as seen in the plane of the form diagram.

\subsection{Numerical integration}

In this work, a standard Gauss quadrature was used to numerically compute the integrals over the surfaces and curves $^{45}$. Because the stress components are calculated from a stress function that is not a polynomial in general, a sufficient number of integration points is hard to estimate. Hence, the number of integration points was controlled by an integration multiplier, denoted NI.

Denote the number of control points in the $u$ and $v$ directions by NU respectively NV and the degree of the NURBS surface by Dim. Then a NURBS surface is typically divided into $(\mathrm{NU}-\mathrm{Dim}) \times(\mathrm{NV}-\mathrm{Dim})$ smaller patches, and each of them is dependent on $(\operatorname{Dim}+1) \times(\operatorname{Dim}+1)$ control points ${ }^{59}$.

In this study, $\left(\operatorname{Dim} \times \operatorname{Dim} \times(\mathrm{NI})^{2}\right)$ Gauss integration points were distributed on each patch (i.e. $n=D i m \times N I$ in the commonly used Gaussian quadrature rule tabulation is used). NURBS surfaces with $\operatorname{Dim}=3$ and $\mathrm{NI}=1$ or 2 works for most cases, resulting in $\mathrm{n}=3$ or 6 for the Gauss quadrature rule.

\subsection{Convergence study}

A variational operator $\delta$ has infinite degrees of freedom (DoF) by definition. The Galerkin method restricts the variational operator to a finite number of DoF equal to the DoF of the finite element model. If a solution exists in the original PDE, by increasing the DoF in the system, the numerical solution should converge to the exact solution.

In this study, the convergence towards the exact solution is evaluated by studying the total energy of the system given by

$$
E=\sum_{\text {surfaces }} \frac{1}{2} \int \bar{S}^{i j} h_{i j} \mathrm{~d} \bar{a}+\sum_{\text {curves }} \frac{1}{2} \int \bar{n}^{11} h_{11} \mathrm{~d} \bar{\ell} .
$$

The elastic energy of the springs are omitted because they will have high energy if used as hard constraints.

It is known that there are incompatible boundary conditions (BCs) that make hyperbolic PDEs ill-posed (i.e. solutions do not exist). In those cases, numerical solutions hardly ever converge. Since a mix of tension and compression stresses results in a hyperbolic PDE, attention must be paid to such situations since the proposed numerical method returns a solution regardless of or not. A guideline to choose proper BCs is provided in Section 4.

\section{Benchmark problems}

\subsection{Hypar}

Consider a simple stress function

$$
\phi=-\frac{1}{2} x^{2}+\frac{1}{2} y^{2},
$$

that is defined on a square that spans between $(-1,-1)$, $(1,-1),(1,1)$, and $(-1,1)$. In Figure 9, the purple surface corresponds to $\phi$ and the gray flat panels are the stress-free region outside the structure apart from the reaction forces seen as the kinks at the top.

The force diagram of $\phi$ is given by $(X, Y)=(-x, y)$, which is curl-free. Figure 10 depicts the force diagram and the 'jumps' of the normal vectors between the smooth pink surface of $\phi$ and the surrounding flat gray planes. By adding edge beams, the shell can be supported by only two points, and the magnitudes of the 'jumps' represent the axial forces in the beams.
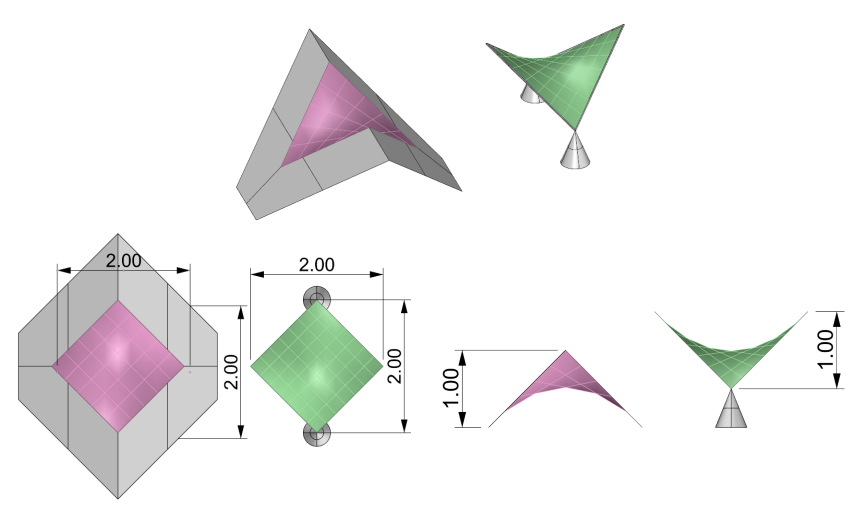

Figure 9. Stress function (purple) and corresponding shell (green) of the hypar problem.

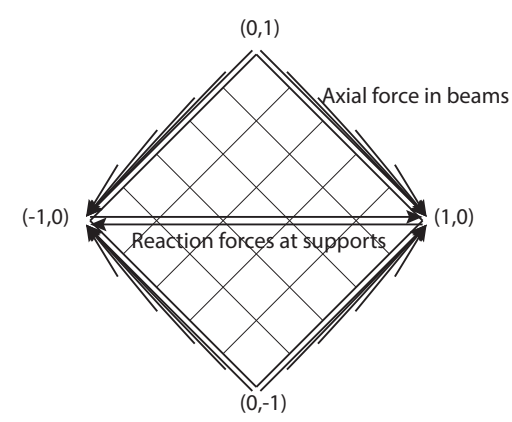

Figure 10. Force diagram and the 'jumps' of the hypar problem.

Substituting Eq. (33) into Eq. (1) gives

$$
-\frac{\partial^{2} z}{\partial y^{2}}+\frac{\partial^{2} z}{\partial x^{2}}=-\rho
$$


for which there exists a known solution,

$$
z=\frac{\rho}{4}\left(x^{2}-y^{2}\right)
$$

The solution satisfies the boundary equilibrium between the edge beams and the shell.

Note that Eq. (35) is only a particular solution. With more complex BCs, the solution is a sum of the particular solution and a general solution (a solution of Eq. (34) where the right-hand side is set to zero) that satisfies the BCs. While a general solution always exists in an elliptical problem, in a hyperbolic problem, there exist incompatible BCs where general solutions do not exist. In general, a hyperbolic PDE only accepts compatible BCs, otherwise the problem becomes ill-posed. This issue is further addressed in Section 4.

This problem can be used as a useful benchmark problem, and the proposed method was tested. The green surface in Figure 9 represents the solution obtained using the proposed method with $\rho=4.0$ as load. The height of the obtained green shell is $1 / 4$ of $\rho$, which matches Eq. (35).

The blue surface in Figure 11 is a plot of the left-hand side of Eq. (1) multiplied by $1 / \rho$ obtained numerically with a NURBS surface with $32 \times 32$ control points, Dim $=3$, and integration point multiplier NI $=1$. It clearly shows a drop by -1 from the red surface representing zero loading. This indicates the method provides the sought solution.

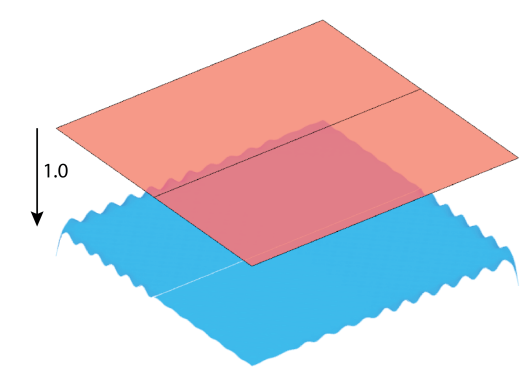

Figure 11. Left-hand side of vertical equilibrium obtained using $32 \times 32$ control points (blue surface) and zero-loading reference (red surface) for the hypar problem.

Figure 12 shows a convergence analysis of this example. As shown in the figure, the total energy remains flat even if the number of control points were raised high. This is because the hypar can be accurately represented with a NURBS surface of $2 \times 2$ control points and with Dim $=3$.

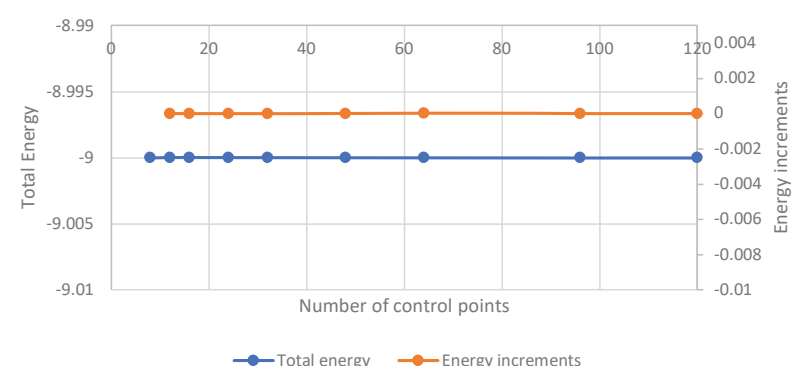

Figure 12. Convergence analysis of the hypar problem.

\subsection{Bowl}

Consider a stress function $\phi$ and a shell $z$ that both have a radial symmetry defined in a polar coordinate system. Then the vertical equilibrium becomes

$$
\frac{1}{r}\left(\frac{\partial \phi}{\partial r} \frac{\partial^{2} z}{\partial r^{2}}+\frac{\partial^{2} \phi}{\partial r^{2}} \frac{\partial z}{\partial r}\right)=-\rho,
$$

where $r \geq C \geq 0$ is the parameter in the radial direction in the polar coordinate system.

By assuming the same shape for the shell and stress function, so that $\phi(r)=A z(r)$ where $A$ is a constant, the solution to Eq. (36) is given by

$$
z(r)=\frac{1}{2 \sqrt{2 A}}\left(r \sqrt{B^{2}-r^{2}}+\arcsin r\right),
$$

with $r \leq B \leq 1$. Thus, at the circumference where $r=B$, concentrated stresses parallel to the circumference direction. If vertical support is provided at $r=C$, then the edge at $r=$ $B$ can 'float' without any support and the circumferential concentrated stress is tensile.

Let $A=1 / 2, B=1, C=0.2$, and $\rho=1$. Then the total height of the shell is given from the analytical solution as $z(B)-z(C)=z(1.0)-z(0.2)=0.5867$ and the cross section of the shell is plotted in Figure 13. Solving $z$ numerically with the proposed method gives the shape shown in Figure 14 which profile and total height 0.58 match the analytical solution.

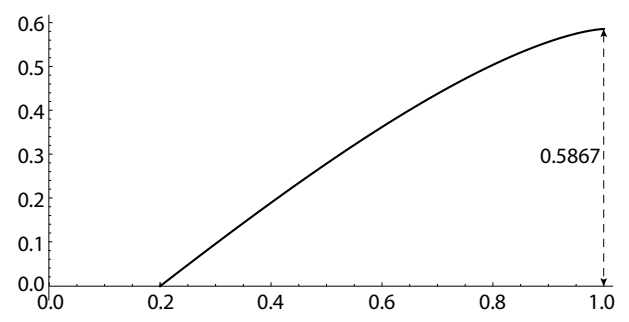

Figure 13. Cross section of analytical solution of the bowl problem.

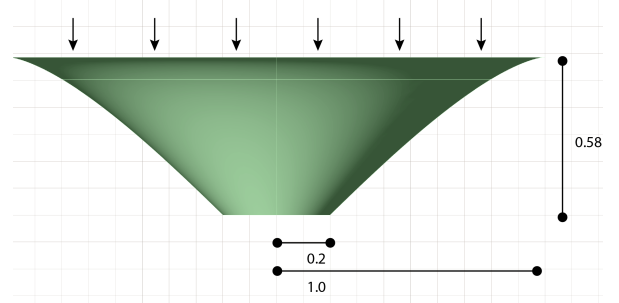

Figure 14. Elevation of numerical solution of the bowl problem.

The blue surface in Figure 15 is a plot of the left-hand side of Eq. (36) multiplied by $1 / \rho$ obtained numerically with a NURBS surface with $32 \times 32$ control points, $\operatorname{Dim}=3$, and integration point multiplier NI $=1$. Similarly as in the hypar example, the blue surface sits at a level -1 below the red surface representing zero loading, again indicating the method provides the sought solution.

A convergence analysis was also conducted. Figure 16 shows plots of the energy as a function of the number of control points, proving the solution converges to a single solution. 


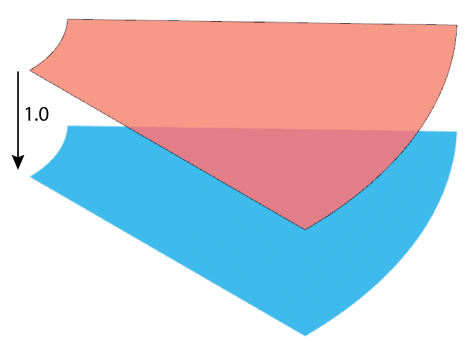

Figure 15. Left-hand side of vertical equilibrium obtained using $120 \times 60$ control points (blue surface) and zero-loading reference (red surface) for the bowl problem.

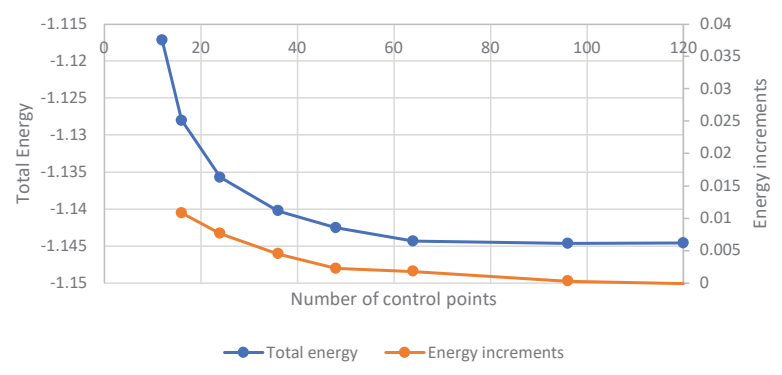

Figure 16. The convergence of the total energy of the bowl problem.

Unlike pure-compression or pure-tension zones, there is a restriction to the boundary conditions in tensioncompression mixed zones. In this example, because we assumed a radial symmetry when deriving the stress function, fixing the rim of the oculus along a flat circle should work, i.e. it is compatible to the problem. Although there might be other compatible boundary conditions not yet identified, but any incompatible boundary condition will make the problem ill-posed.

As such, in general, a hyperbolic partial differential equation is well-posed only when a proper set of compatible boundary conditions are given. This makes solving the hyperbolic problems substantially different from solving elliptic problems, i.e. pure-compression or puretension ones. Next section further elaborates the topic about compatible and incompatible boundary conditions of hyperbolic PDEs.

\section{Asymptotic lines and boundary conditions}

Within areas of pure compression or tension, support boundary conditions (BCs) cause singularities that dissipate rapidly and only affect the solution locally, allowing free placement of the supports in such areas. However, in areas with a mix of compression and tension, $\mathrm{BCs}$ give rise to singularities that transfer through characteristic lines with no dissipation, often making the hyperbolic PDE ill-posed ${ }^{5,60}$. Such incompatible BCs should be avoided.

The four green surfaces in Figure 17a are the numerical solutions obtained by imposing two compatible sets of BCs and two incompatible ones on the hypar example introduced in Section 3.1. As in Figures 11 and 15, the blue surfaces of Figure $17 \mathrm{a}$ are supposed to drop by 1.0 below the reference surface if the PDE is well-posed. However, the incompatible
BCs cause disturbances seen as bumpy ridges that run across the solution, and the blue surfaces do not drop by 1.0 but form spikes.

A second-order hyperbolic PDE is often called a wave equation, and waves propagate along so-called characteristic lines. Thus, a perturbance transfers from one end of a characteristic line to the other end with no dissipation, and if no compatible $\mathrm{BC}$ exists at the other end, the problem becomes ill-posed (i.e. no solution exists). Even if solutions do not exist, the proposed method returns a numerical solution. Hence, extra attention must be paid to this issue.

In the problems discussed in this paper, the characteristic lines are the projections of the asymptotic lines of the stress function to the ground plane (see Chapter 4 in Csonka $^{5}$, Chapters 1 and 2 in Sanchez-Palencia et al. ${ }^{61}$, and Appendix C). An asymptotic line is a line on a surface whose normal curvature is zero, and it only exists in areas where the Gaussian curvature is negative. Typically, the asymptotic lines are a group of diagonal lines that run across the negative Gaussian curvature area, intersecting the principle curvature lines at roughly 45 degrees.

The general solution of the hypar problem is $z(x, y)=$ $F(x+y)+G(x-y)$ with $F$ and $G$ arbitrary functions that are determined such that boundary conditions are satisfied, and the asymptotic lines are such that they result in characteristic lines given by $x-y=$ const. and $x+y=$ const., which run parallel with the edges of the shell. As seen in Figure 17a, the BCs that contradict the general solution are incompatible or, in other words, BCs that do not have compatible $\mathrm{BCs}$ at the end of the characteristic lines are incompatible. Figures $17 \mathrm{~b}$ and $17 \mathrm{c}$ show more examples of compatible and incompatible BCs imposed on the bowl example of Section 3.2 respectively on a problem reproducing Félix Candela's Xotimilco restaurant (see Appendix D). The BCs for these problems were guessed based on the knowledge of the radial symmetry. However, in general, identifying compatible BCs for hyperbolic PDEs is a challenge.

To avoid this challenge, 'safe' areas with positive Gaussian curvature may be added to the stress function in which the supports are placed. This strategy is illustrated in the examples in Figure 18 where the purple surfaces are the used Airy stress functions with their asymptotic lines drawn in white. Though not shown, the 'blue surfaces' dropped properly for all cases. The safe-area-strategy is used on the examples discussed in Section 5.

\section{More complex example}

\subsection{A stress function that can take a general plan geometry}

Given a closed flat guide curve defined by $\left(c_{x}(u), c_{y}(u)\right)$, a simple pair of stress function $\phi$ and form diagram $\boldsymbol{x}$ may be obtained as

$$
\left.\begin{array}{l}
\phi(u, v)=Q(v) \\
\boldsymbol{x}(u, v)=\left(v c_{x}(u), v c_{y}(u)\right)
\end{array}\right\}
$$

where $v$ is bounded by $v_{\min }>0$ and $v_{\max }$, and $Q(v)$ defines the profile of the stress function. Then, from Eq. (10), the 


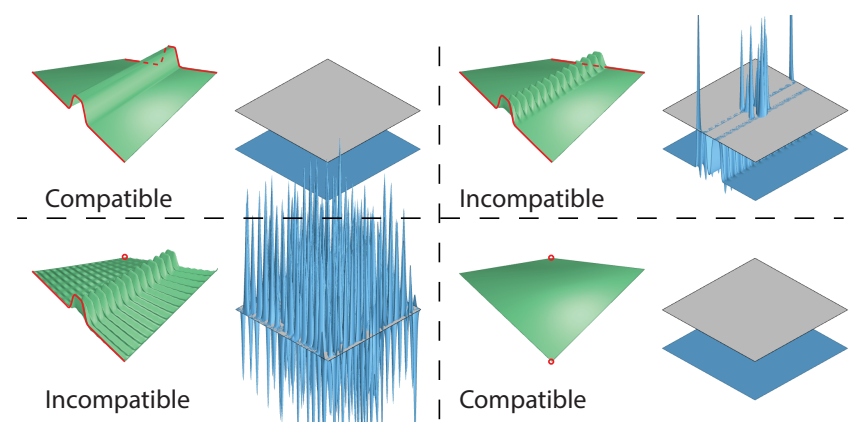

(a) Hypar example of Section 3.1.

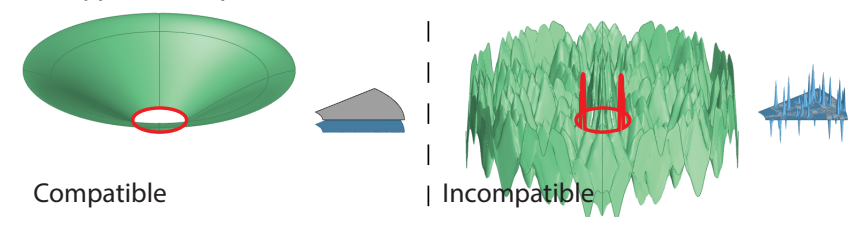

(b) Bowl example of Section 3.2.

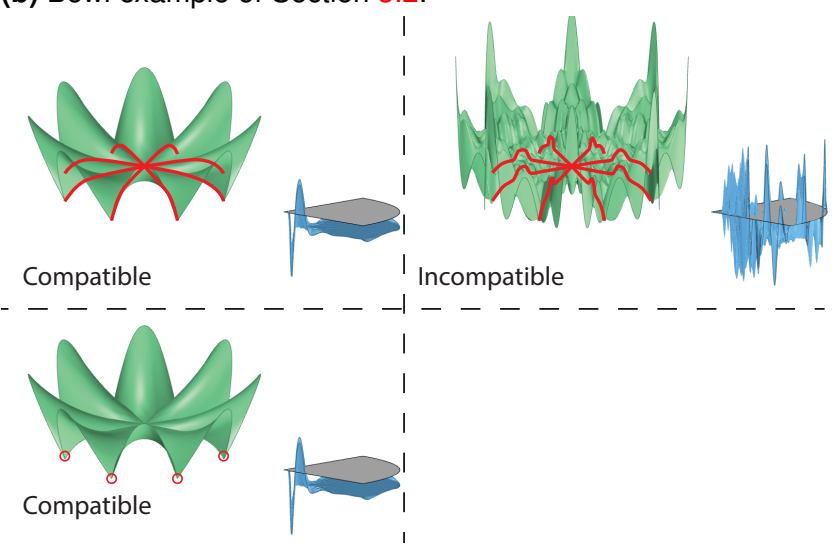

(c) Félix Candela example briefly mentioned in Appendix D.

Figure 17. Compatible and incompatible BCs: BCs (red), numerical solution (green), and left-hand-side of equilibrium multiplied by $1 / \rho$ (blue), which for proper solutions should drop by 1.0 below the references surface (gray).

force diagram follows as

$$
\boldsymbol{X}=Q^{\prime}(v) \frac{1}{c_{x} c_{y}^{\prime}-c_{y} c_{x}^{\prime}}\left(c_{y}^{\prime},-c_{x}^{\prime}\right),
$$

where a prime is used to denote differentiation.

If $Q^{\prime}(v)=0$ at an edge of the shell, the stress function tangentially touches the horizontal plane intersecting the edge of the stress function. Such points on the form diagram $\boldsymbol{x}$ are mapped to the center of the force diagram $\boldsymbol{X}$. Therefore, the distances between any points of the edge as measured in the force diagram is always zero. Hence, there is no horizontal thrust acting on the edge. On the other hand, if $Q^{\prime}(v) \neq 0$ at an edge, the stress function forms a kink with the horizontal plane intersecting the edge.

As pointed out before, for example, when discussing the kinks between the hypar stress function and the surrounding planes in Figure 10, a kink represent concentrated stresses acting along the edge, which are balanced by stresses acting in the perpendicular direction. The magnitude of the axial force can be obtained by measuring the 'jump' from the corresponding points of the edge in the force diagram to its center, which represent the horizontal-thrust acting on the edge.

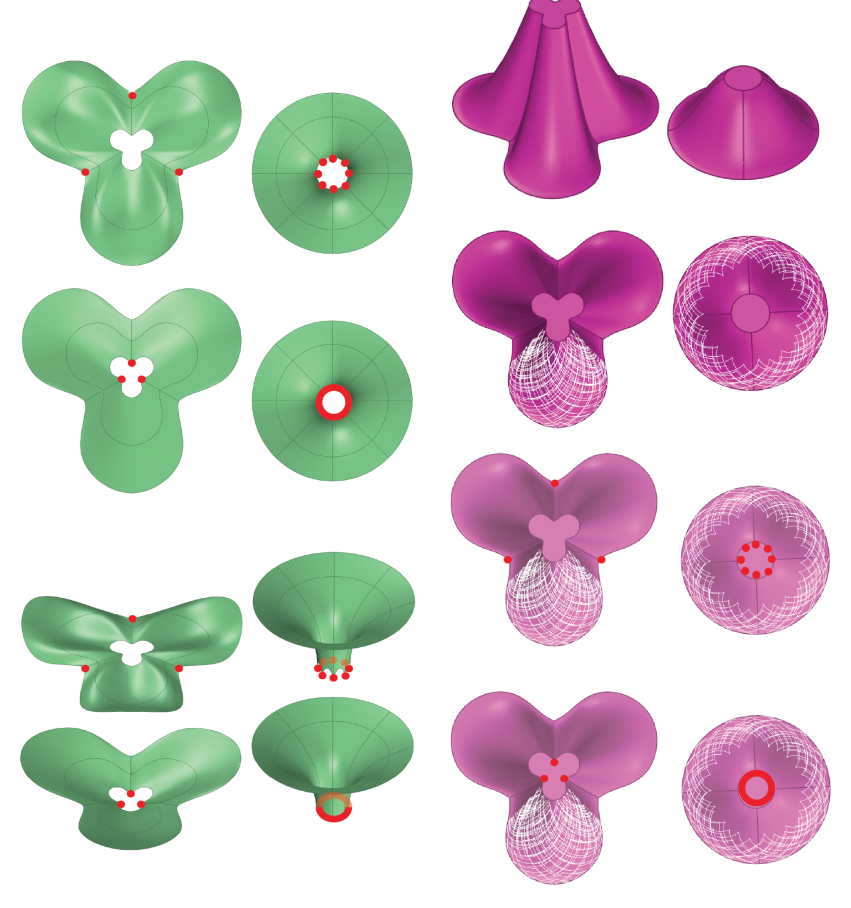

Figure 18. Putting supports away from the asymptotic line zones is recommended to avoid the compatibility issue of BCs. (green): shells, (purple): stress functions.

The proposed stress function, form diagram, and force diagram of Eqs. (38) and (39) are valid not only for smooth guide curves, but also for piecewise smooth guide curves. In those cases, the stress function also becomes a piecewise smooth function, with creases along lines intersecting the discontinuities in the guide curve. As discussed in Section 2.7, such stress functions results in 'jumps' in the force diagram representing concentrated axial forces running along the corresponding creases in the shell, and their magnitudes are given by Eq. (29).

Figure 19 shows a form diagram generated using a piecewise smooth guide curve and $v_{\min }=0.2, v_{\max }=1.0$. Vertical supports are provided along three short stretches of the exterior perimeter of the form diagram. Along these stretches, the form diagram is modified with small semicircles that ensure a local area of pure compression close to the support avoiding problems of incompatible BCs.

Let the stress function $\phi=Q(v)$ be such that

$$
Q^{\prime}(v)=(v-1)+12 \lambda(v-1)^{2},
$$

where $\lambda$ is a parameter embedded to the equation to control the speed of the scaling of the guide curve. Then a stress function that comprises three smooth areas and three crease curves is obtained shown to the left in Figure 20, resulting in a force diagram shown to the right. As illustrated in Figure 20, if one travels from point $a$ to $b$ to $c$ on the stress function, a corresponding travel from $A$ to $B$ to $C$ takes place on the force diagram where point $B$ exist on both sides of the 'jump' representing the crease running through point $b$ on the stress function.

Figure 21 shows two numerical form-finding results obtained using the discussed functions $\phi, \boldsymbol{x}$, and $\boldsymbol{X}$, one using the linear method (Sec. 2.4) and the other the nonlinear method (Sec. 2.5). Each leaf is represented by 2 NUBRS 
surfaces with $32 \times 16$ control points, $\operatorname{Dim}=3, \mathrm{NI}=2$, and $\lambda$ of Eq. (40) was set to 0.

The resulting shells are rather similar, with the nonlinear shell a little taller than the linear shell. The difference is due to the accurate account of the surface area in the nonlinear method. The shells were obtained with loading $\rho=-0.05$ and the nonlinear method converged as proved by the history of the residual norm shown in Figure 22. However, when the load was increased to $\rho=-0.15$, the nonlinear method did not converge. As such, the nonlinear method does not converge when the loading is big. However, fortunately, in the cases we tested, although it depends on the problems, the height of the shell at the maximum loading $\rho$ that gave a convergence gives forms that are usable as architectural spaces.

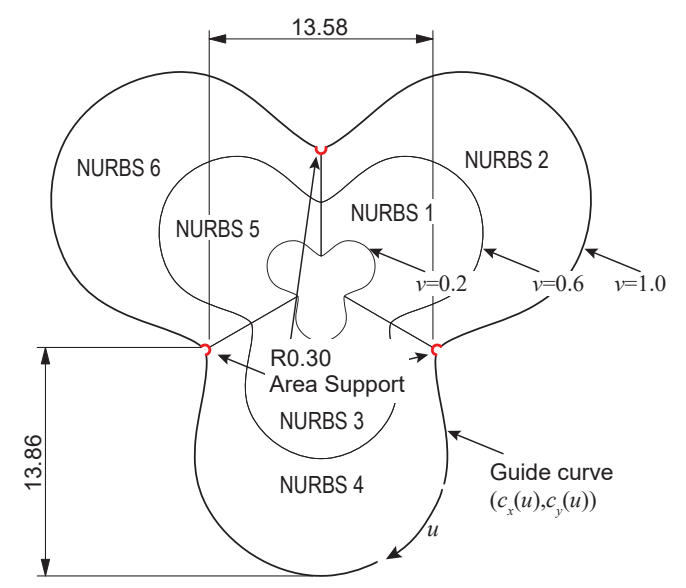

Figure 19. Dimensions of the used form diagram (unit=[m]).
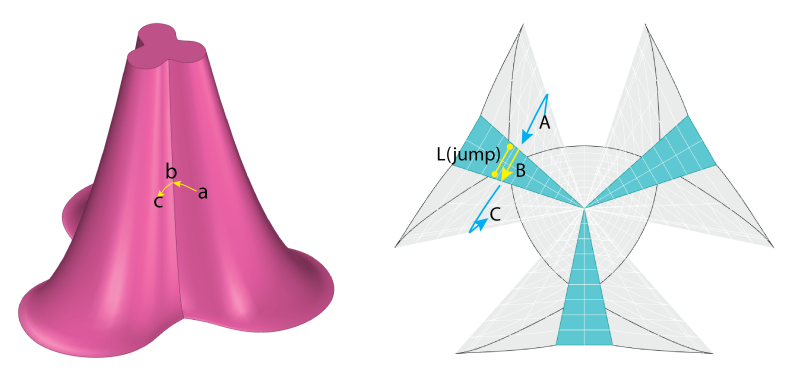

Figure 20. A piecewise-smooth stress function (left) and the corresponding a force diagram (right).
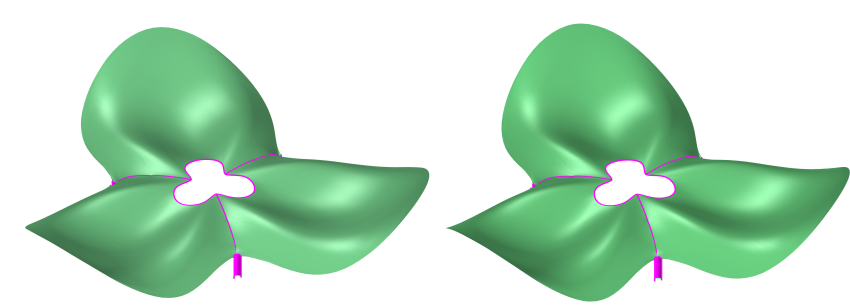

Figure 21. Solutions using the linear method (left) and the nonlinear method (right), both with $\rho=-0.05$.

\subsection{Verification}

A convergence study was conducted by increasing the number of control points from $20 \times 10$ to an extreme of

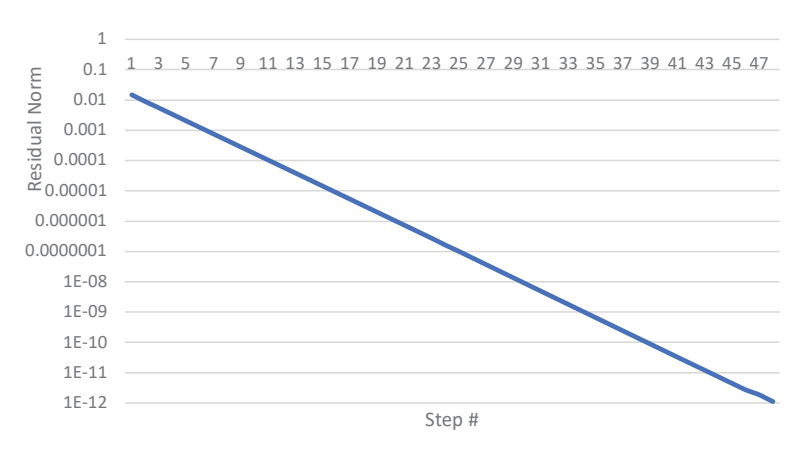

Figure 22. History of the residual norm of the vertical equilibrium equation solved with nonlinear method and $\rho=-0.05$.

$400 \times 200$. Dim $=3$ NURBS surfaces were used and the integration point multiplier was set to $\mathrm{NI}=2$. As shown in Figure 23, the solutions were very stable even if the number of control points were raised. Figure 24 is a plot of the energy versus the number of control points, and the solutions rapidly converged.

So far, in the form-finding process, only an ideal membrane stress state is considered, and thus, structural properties such as thickness, stiffness and deformation are not studied.

Using the shell obtained from the nonlinear method, a series of deformation analyses were performed by varying the thickness of the shell from a realistic one to an extremely small one. The three crease curves where modeled as solid beams with cross-section width $\times$ height $=0.1 \mathrm{~m} \times 0.2 \mathrm{~m}$, and standard steel was used for both the shell and beams. The analysis was performed using NURBS-based shell and beam elements provided by Kiwi3 ${ }^{62}$, which consider both membrane and bending stiffness. The NURBS surfaces and curves obtained in the form-finding stage was used as finite elements without meshing.

Table 1 shows the maximum deflection with the varied thickness. Even for the small thicknesses, the deformations are kept rather low. This indicates that the shell is working primarily in membrane action, just as intended.

Another way to check if the shell works in pure membrane action is to study the ratio between the membrane energy and the bending energy of the shell. As can be seen in Figure 25, the membrane energy becomes more dominant as the thickness of the shell is decreased.

Figure 26 displays the left hand side of the vertical equilibrium multiplied with $1 \rho$ obtained for a third of the shell with $240 \times 120$ control points, $\operatorname{Dim}=3, \mathrm{NI}=2$. As expected, the blue surface drops by 1.0 apart from at two areas which corresponds to the location of the supports.

From these observations, it can be concluded that the obtained solution is an approximation of the original equilibrium equation and that the shell works by membrane action.

Remarkably, a few of the asymptotic lines of the stress function (Fig. 27, left) emerge as waves running on the formfinding result (Fig. 27, right). Compared to the bumpy ridges in the numerical solutions obtained with incompatible BCs discussed in section 4 , these waves look clean, and there are 
Table 1. Maximum deflection with varied thickness. Percentage based on cantilever depth $L=13860 \mathrm{~mm}$.

\begin{tabular}{ccc}
\hline $\begin{array}{c}\text { Thickness } \\
t(\mathrm{~mm})\end{array}$ & $\begin{array}{c}\text { Max deflection } \\
\delta(\mathrm{mm})\end{array}$ & $\begin{array}{c}\text { Deflection/cantilever depth } \\
\delta / L(\%)\end{array}$ \\
\hline 0.1 & 76 & 0.55 \\
0.5 & 80 & 0.57 \\
1 & 77 & 0.55 \\
5 & 54 & 0.39 \\
10 & 40 & 0.28 \\
50 & 21 & 0.15 \\
\hline
\end{tabular}

no spiky noises in the blue surface except the areas near the supports. Thus, those waves can be distinguished from the numerical errors caused by the incompatible BCs, rather, it can be considered proving that a solution of a wave equation is obtained.
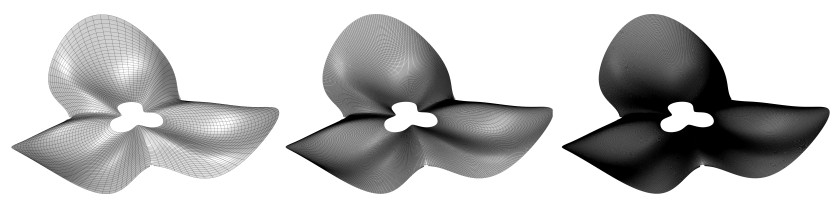

Figure 23. From left to right: solutions obtained with $40 \times 20$, $160 \times 80,360 \times 180$ control points.

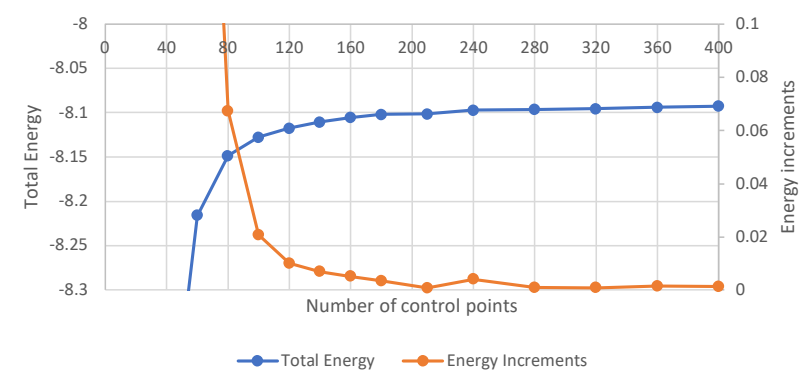

Figure 24. Convergence study, (blue) total energy, (orange) increments of the energy from the last step..

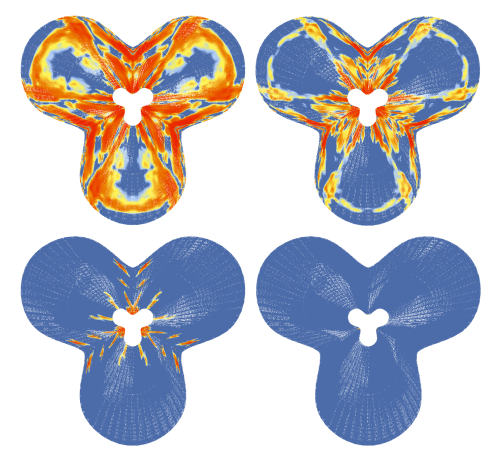

Figure 25. Ratio between membrane energy and bending energy for thicknesses $10,5,1$, and $0.1 \mathrm{~mm}$. Blue $=100 \%$ membrane energy and red $=100 \%$ bending energy.

\subsection{Implementation scenario}

Figure 28 shows an architectural rendering that illustrates an implementation scenario of the example.

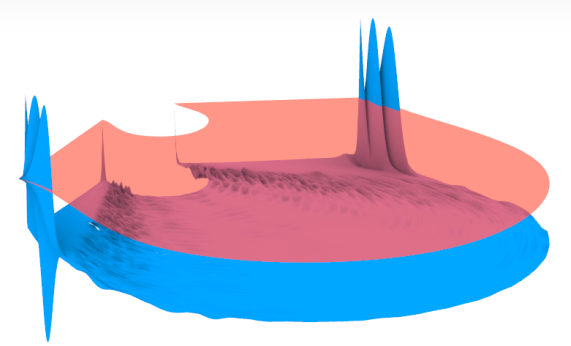

Figure 26. A blue surface obtained with $240 \times 120$ control points, $\operatorname{Dim}=3$, and NI $=2$.
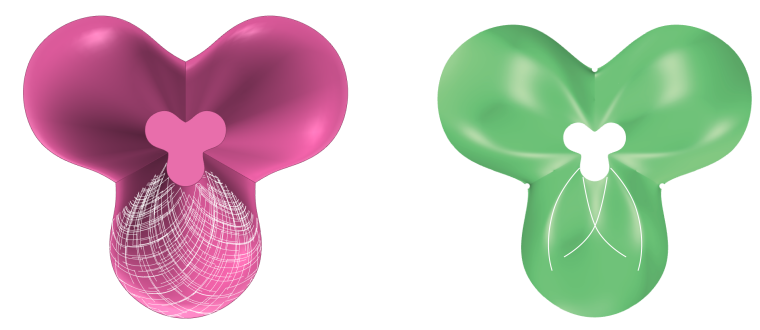

Figure 27. An overlay of asymptotic lines (left) to the obtained solution (right).

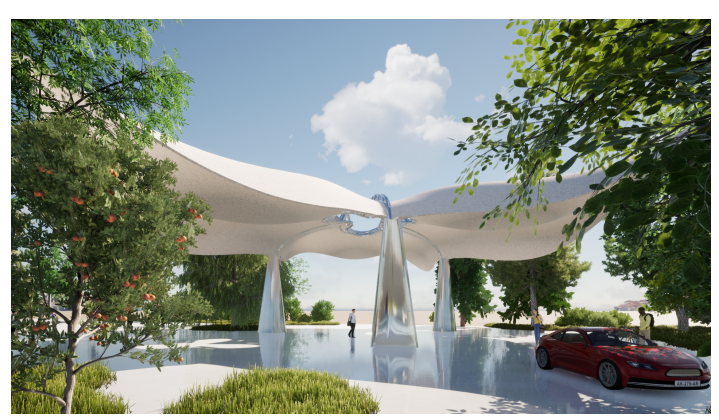

Figure 28. An architectural implementation scenario of the example discussed in this section.

\section{Conclusion}

The numerical form-finding of membrane action shells containing a mix of tensile and compressive stresses involves solving a hyperbolic second-order partial differential equation. In this paper, an existing NURBS-based isogeometric approach originally designed for the form-finding of pure compression or pure tension membrane shells was developed further. It is demonstrated that the developed method can be used to solve tension-compression mixed type hyperbolic equilibrium equations with satisfactory accuracy.

The method takes as input a form diagram and a stress function from which a force diagram is computed, and their reciprocal relations are discussed.

In areas where the stress function describes a state of both tension and compression, it has negative Gaussian curvature and thus asymptotic lines, and it was recommended to put supports away from such areas. Otherwise, the boundary conditions must be compatible with the equilibrium equation to make the problem well-posed.

The proposed method was tested using simple problems whose analytical solutions are known, and the numerical and analytical results were shown to have a good agreement. A solution to a more complex problem was also demonstrated 
and verified, and the formulation of the problem can easily be modified to suit a wide range of architectural applications

\section{Acknowledgements}

This research was supported by Skidmore, Owings and Merrill from 2015 to 2020 .

\section{Declaration of conflicting interests}

The Author(s) declare(s) that there is no conflict of interest.

\section{Funding}

This research received no specific grant from any funding agency in the public, commercial, or not-for-profit sectors.

\section{Supplemental material}

The test codes are published to the public at http://mikity . wikidot.com/kingghidorah. The codes run in Rhinoceros 6® and Grasshopper $\AA$ environment.

\section{References}

1. Miki M, Igarashi T and Block P. Parametric self-supporting surfaces via direct computation of Airy stress functions. ACM Transactions on Graphics (TOG) 2015; 34(4): 1-12.

2. Miki M, Mitchell $\mathrm{T}$ and Baker W. Continuum form and force diagrams in NURBS-based shell form-finding. In Lázaro C, Bletzinger KU and Oñate E (eds.) Form and Force 2019. International Association for Shell and Spatial Structures (IASS), pp. 1-8.

3. Airy GB. IV. On the strains in the interior of beams. Philosophical Transactions of the Royal Society of London 1863; 153: 49-79. DOI:10.1098/rstl.1863.0004.

4. Green AE and Zerna W. Theoretical elasticity. Second edition ed. Oxford University Press, 1968. ISBN 0486670767.

5. Csonka P. Theory and practice of membrane shells. tpms 1987;

6. Timoshenko SP and Woinowsky-Krieger S. Theory of plates and shells. McGraw-hill, 1959.

7. Pucher A. Die berechnung der dehnungsspannungen von rotationsschalen mit hilfe von spannungsfunktionen. Int Vereinig Brücken-u Hochbau Abh 1938; 5: 275.

8. Williams $\mathrm{C}$ and McRobie A. Graphic statics using discontinuous Airy stress functions. International Journal of Space Structures 2016; 31(2-4): 121-134.

9. Klein $\mathrm{F}$ and Wieghardt $\mathrm{K}$. Über spannungsflächen und reziproke diagramme mit besonderer berücksichtiging der maxwellschen arbeiten. Arch Math Phys III/VIII 1904;

10. Crapo $\mathrm{H}$ and Whiteley W. Statics of frameworks and motions of panel structures: a projective geometric introduction. Structural Topology, 1982, núm 6 1982; .

11. Fraternali F, Angelillo $M$ and Fortunato A. A lumped stress method for plane elastic problems and the discrete-continuum approximation. International journal of solids and structures 2002; 39(25): 6211-6240.

12. Fraternali F. A thrust network approach to the equilibrium problem of unreinforced masonry vaults via polyhedral stress functions. Mechanics Research Communications 2010; 37(2): 198-204.

13. Fraternali $F$ and Carpentieri G. On the correspondence between $2 \mathrm{D}$ force networks and polyhedral stress functions.
International Journal of Space Structures 2014; 29(3): 145159.

14. Vouga E, Höbinger M, Wallner $\mathrm{J}$ et al. Design of selfsupporting surfaces. ACM Transactions on Graphics (TOG) 2012; 31(4): 1-11.

15. Varignon P. Nouvelle Mecanique Ou Statique: Dont Le Projet Fut Donné En M. DC. LXXXVII, volume 2. Jombert, 1725.

16. Maxwell JC. Xlv. on reciprocal figures and diagrams of forces. The London, Edinburgh, and Dublin Philosophical Magazine and Journal of Science 1864; 27(182): 250-261.

17. Culmann C. Die graphische statik, volume 1. Meyer \& Zeller, 1875.

18. Rankine WM. Xvii. principle of the equilibrium of polyhedral frames. The London, Edinburgh, and Dublin Philosophical Magazine and Journal of Science 1864; 27(180): 92-92.

19. Cremona L. Le figure reciproche nella grafica statica. Tipografia Giuseppe Bernardoni, Milano 1872;

20. Cremona L. Le figure reciproche nella statica grafica. Hoepli, 1879.

21. Micheletti A. On generalized reciprocal diagrams for selfstressed frameworks. International Journal of Space Structures 2008; 23(3): 153-166.

22. Zanni G and Pennock GR. A unified graphical approach to the static analysis of axially loaded structures. Mechanism and machine theory 2009; 44(12): 2187-2203.

23. Van Mele T and Block P. Algebraic graph statics. ComputerAided Design 2014; 53: 104-116.

24. Lee J, Mueller C and Fivet C. Automatic generation of diverse equilibrium structures through shape grammars and graphic statics. International Journal of Space Structures 2016; 31(24): 147-164.

25. Lee J, Van Mele T and Block P. Disjointed force polyhedra. Computer-Aided Design 2018; 99: 11-28.

26. Mazurek A, Beghini A, Carrion J et al. Minimum weight layouts of spanning structures obtained using graphic statics. International Journal of Space Structures 2016; 31(2-4): 112120.

27. Mitchell T, Baker W, McRobie A et al. Mechanisms and states of self-stress of planar trusses using graphic statics, part I: The fundamental theorem of linear algebra and the airy stress function. International Journal of space structures 2016; 31(24): 85-101.

28. Alic V and Åkesson D. Bi-directional algebraic graphic statics. Computer-Aided Design 2017; 93: 26-37.

29. Baranyai T. On the admissible dualities in Maxwell's reciprocal construction. International Journal of Solids and Structures 2019; 171: 10-16.

30. McRobie A. The geometry of structural equilibrium. Royal Society open science 2017; 4(3): 160759.

31. Akbarzadeh M, Van Mele T and Block P. On the equilibrium of funicular polyhedral frames and convex polyhedral force diagrams. Computer-Aided Design 2015; 63: 118-128.

32. Fivet C. Projective transformations of structural equilibrium. International Journal of Space Structures 2016; 31(2-4): 135146.

33. Ole Ohlbrock P and Schwartz J. Combinatorial equilibrium modeling. International Journal of Space Structures 2016; 31(2-4): 177-189.

34. Ohlbrock PO and D'Acunto P. A computer-aided approach to equilibrium design based on graphic statics and combinatorial variations. Computer-Aided Design 2020; 121: 102802. 
35. McRobie A. Maxwell and Rankine reciprocal diagrams via Minkowski sums for two-dimensional and three-dimensional trusses under load. International Journal of Space Structures 2016; 31(2-4): 203-216.

36. Konstantatou M, D'Acunto P and McRobie A. Polarities in structural analysis and design: n-dimensional graphic statics and structural transformations. International Journal of Solids and Structures 2018; 152: 272-293.

37. Bolhassani M, Akbarzadeh M, Mahnia M et al. On structural behavior of a funicular concrete polyhedral frame designed by 3D graphic statics. In Structures, volume 14. Elsevier, pp. 5668.

38. D'acunto P, Jasienski JP, Ohlbrock PO et al. Vector-based 3D graphic statics: A framework for the design of spatial structures based on the relation between form and forces. International Journal of Solids and Structures 2019; 167: 58-70.

39. Block P and Ochsendorf J. Thrust network analysis: a new methodology for three-dimensional equilibrium. Journal of the International Association for shell and spatial structures 2007; 48(3): 167-173.

40. Liu Y, Pan H, Snyder J et al. Computing self-supporting surfaces by regular triangulation. ACM Transactions on Graphics (TOG) 2013; 32(4): 1-10.

41. Rippmann M, Lachauer L and Block P. Interactive vault design. International Journal of Space Structures 2012; 27(4): 219230.

42. Rippmann M and Block P. Funicular funnel shells. In Rethinking prototype. Proceedings of the Design Modelling Symposium. Berlin. pp. 75-89.

43. De Goes F, Alliez P, Owhadi $\mathrm{H}$ et al. On the equilibrium of simplicial masonry structures. ACM Transactions on Graphics (TOG) 2013; 32(4): 1-10.

44. Panozzo D, Block P and Sorkine-Hornung O. Designing unreinforced masonry models. ACM Transactions on Graphics (TOG) 2013; 32(4): 1-12.

45. Hughes TJ, Cottrell JA and Bazilevs Y. Isogeometric analysis: CAD, finite elements, NURBS, exact geometry and mesh refinement. Computer methods in applied mechanics and engineering 2005; 194(39-41): 4135-4195.

46. Kiendl J, Bletzinger KU, Linhard J et al. Isogeometric shell analysis with Kirchhoff-Love elements. Computer Methods in Applied Mechanics and Engineering 2009; 198(49-52): 39023914.

47. Breitenberger M, Apostolatos A, Philipp B et al. Analysis in computer aided design: Nonlinear isogeometric B-Rep analysis of shell structures. Computer Methods in Applied Mechanics and Engineering 2015; 284: 401-457.

48. Bauer AM, Wüchner R and Bletzinger KU. Innovative CADintegrated isogeometric simulation of sliding edge cables in lightweight structures. Journal of the International Association for Shell and Spatial Structures 2018; 59(4): 251-258.

49. Allen E and Zalewski W. Form and forces: designing efficient, expressive structures. John Wiley \& Sons, 2009.

50. Bonet J and Wood RD. Nonlinear continuum mechanics for finite element analysis. Cambridge university press, 1997.

51. Cartan H. Differential forms. Courier Corporation, 2006.

52. Eisenhart LP. Riemannian geometry, volume 51. Princeton university press, 1997.

53. Ciarlet PG. Mathematical Elasticity: Volume I: threedimensional elasticity. North-Holland, 1988.
54. Ciarlet PG. Mathematical Elasticity: Volume II: Theory of Plates. Elsevier, 1997.

55. Ciarlet PG. An introduction to differential geometry with applications to elasticity. Journal of Elasticity 2005; 78(1-3): 1-215.

56. Bletzinger KU, Wüchner R, Daoud F et al. Computational methods for form finding and optimization of shells and membranes. Computer methods in applied mechanics and engineering 2005; 194(30-33): 3438-3452.

57. Connor JJ and Brebbia CA. Finite element techniques for fluid flow. Newnes, 2013.

58. Pauletti RM and Pimenta PM. The natural force density method for the shape finding of taut structures. Computer Methods in Applied Mechanics and Engineering 2008; 197(4950): 4419-4428.

59. Rogers DF. An introduction to NURBS: with historical perspective. Elsevier, 2000.

60. Colton D. Partial differential equations: an introduction. Courier Corporation, 2004.

61. Sanchez-Palencia E, Millet O and Béchet F. Singular problems in shell theory: computing and asymptotics, volume 54. Springer Science \& Business Media, 2010.

62. Baue A, Längst $\mathrm{P}$, Magna RL et al. Exploring software approaches for the design and simulation of bending active systems. In Mueller C and Adriaenssens S (eds.) Proceedings of the International Association for Shell and Spatial Structures (IASS) Symposium 2018 - Creativity in Structural Design. International Association for Shell and Spatial Structures, pp. 1-8.

\section{A Vector and tensor calculus}

Base vectors and the first fundamental form of a form diagram on a $(u, v)$ system:

$$
\begin{gathered}
\boldsymbol{g}_{u}=\left(\frac{\partial x}{\partial u}, \frac{\partial y}{\partial u}, 0\right)^{T}, \quad \boldsymbol{g}_{v}=\left(\frac{\partial x}{\partial v}, \frac{\partial y}{\partial v}, 0\right)^{T}, \\
\boldsymbol{G}_{u}=\left(\frac{\partial X}{\partial u}, \frac{\partial Y}{\partial u}, 0\right)^{T}, \quad \boldsymbol{G}_{v}=\left(\frac{\partial X}{\partial v}, \frac{\partial Y}{\partial v}, 0\right)^{T}, \\
g_{i j}=\boldsymbol{g}_{i} \cdot \boldsymbol{g}_{j}=\boldsymbol{g}_{i}^{T} \boldsymbol{g}_{j},
\end{gathered}
$$

and calculation of dual base vectors:

$$
\boldsymbol{g}^{u}=g^{u u} \boldsymbol{g}_{u}+g^{u v} \boldsymbol{g}_{v}, \quad \boldsymbol{g}^{v}=g^{v u} \boldsymbol{g}_{u}+g^{v v} \boldsymbol{g}_{v},
$$

where $g^{i j}$ is the inverse of $g_{i j}$. The same relations can be used for a force diagram.

The deformation gradient $F$ is given by Eq. (16) and its components on a $(x, y)$ coordinate system:

$$
\begin{aligned}
& F_{x x}=\left(\boldsymbol{e}_{x} \cdot \boldsymbol{G}_{u}\right)\left(\boldsymbol{e}_{x} \cdot \boldsymbol{g}^{u}\right)+\left(\boldsymbol{e}_{x} \cdot \boldsymbol{G}_{v}\right)\left(\boldsymbol{e}_{x} \cdot \boldsymbol{g}^{v}\right), \\
& F_{x y}=\left(\boldsymbol{e}_{x} \cdot \boldsymbol{G}_{u}\right)\left(\boldsymbol{e}_{y} \cdot \boldsymbol{g}^{u}\right)+\left(\boldsymbol{e}_{x} \cdot \boldsymbol{G}_{v}\right)\left(\boldsymbol{e}_{y} \cdot \boldsymbol{g}^{v}\right), \\
& F_{y y}=\left(\boldsymbol{e}_{y} \cdot \boldsymbol{G}_{u}\right)\left(\boldsymbol{e}_{y} \cdot \boldsymbol{g}^{u}\right)+\left(\boldsymbol{e}_{y} \cdot \boldsymbol{G}_{v}\right)\left(\boldsymbol{e}_{y} \cdot \boldsymbol{g}^{v}\right),
\end{aligned}
$$

where $\boldsymbol{e}_{x}$ and $\boldsymbol{e}_{y}$ the orthonormal base vectors of the $(x, y)$ system.

Base vectors and the first fundamental form on a shell:

$$
\boldsymbol{h}_{\xi}=\left(\frac{\partial x}{\partial \xi}, \frac{\partial y}{\partial \xi}, \frac{\partial z}{\partial \xi}\right)^{T}, \quad \boldsymbol{h}_{\eta}=\left(\frac{\partial x}{\partial \eta}, \frac{\partial y}{\partial \eta}, \frac{\partial z}{\partial \eta}\right)^{T}
$$




$$
h_{i j}=\boldsymbol{h}_{i} \cdot \boldsymbol{h}_{j}=\boldsymbol{h}_{i}^{T} \boldsymbol{h}_{j} .
$$

Those on the projected geometry of a shell:

$$
\begin{gathered}
\overline{\boldsymbol{h}}_{\xi}=\left(\frac{\partial x}{\partial \xi}, \frac{\partial y}{\partial \xi}, 0\right)^{T}, \quad \overline{\boldsymbol{h}}_{\eta}=\left(\frac{\partial x}{\partial \eta}, \frac{\partial y}{\partial \eta}, 0\right)^{T}, \\
\bar{h}_{i j}=\overline{\boldsymbol{h}}_{i} \cdot \overline{\boldsymbol{h}}_{j}=\overline{\boldsymbol{h}}_{i}^{T} \overline{\boldsymbol{h}}_{j} .
\end{gathered}
$$

Components of the 2nd-PK stress tensor measured on $(\xi, \eta)$ system:

$$
\bar{S}^{i j}=\left[\begin{array}{ll}
\frac{\partial x}{\partial \xi} & \frac{\partial y}{\partial \xi} \\
\frac{\partial x}{\partial \eta} & \frac{\partial y}{\partial \eta}
\end{array}\right]\left[\begin{array}{cc}
F_{y y} & -F_{x y} \\
-F_{x y} & F_{x x}
\end{array}\right]\left[\begin{array}{ll}
\frac{\partial x}{\partial \xi} & \frac{\partial x}{\partial \eta} \\
\frac{\partial y}{\partial \xi} & \frac{\partial y}{\partial \eta}
\end{array}\right]
$$

\section{B Principle of virtual work}

The equilibrium equation of a membrane shell:

$$
S_{x x} \frac{\partial^{2} z}{\partial x^{2}}+S_{x y} \frac{\partial^{2} z}{\partial x \partial y}+S_{y y} \frac{\partial^{2} z}{\partial y^{2}}=-\rho .
$$

Defining $\boldsymbol{k}_{1}=\left(1,0, \frac{\partial z}{\partial x}\right), \boldsymbol{k}_{2}=\left(0,1, \frac{\partial z}{\partial y}\right), \boldsymbol{e}_{1}=(1,0,0)$, $\boldsymbol{e}_{2}=(0,1,0)$, and $\boldsymbol{e}_{3}=(0,0,1)$, and rewriting the equation by using a systematic rule, $x, y, z \rightarrow x_{1}, x_{2}, x_{3}$, the LHS of the equilibrium equation can be rewritten as

$$
S_{\alpha \beta} \frac{\partial^{2} z}{\partial x_{\beta} \partial x_{\alpha}}=\frac{\partial S_{\alpha \beta} \boldsymbol{k}_{\alpha} \otimes \boldsymbol{k}_{\beta}}{\partial x_{k}}:\left(\boldsymbol{e}_{3} \otimes \boldsymbol{e}_{k}\right),
$$

where : represents an inner product between two tensors defined by $\boldsymbol{a} \otimes \boldsymbol{b}: \boldsymbol{c} \otimes \boldsymbol{d}=(\boldsymbol{a} \cdot \boldsymbol{d})(\boldsymbol{b} \cdot \boldsymbol{c})$.

Then, when the variation (small change) of the shell is restricted to vertical direction only and represented by $\delta z(x, y)$, the virtual work done by $S_{\alpha \beta}$ is

$$
\begin{aligned}
\delta w= & \text { boundary term } \\
& -\int \partial_{k}\left(S_{\alpha \beta} \boldsymbol{k}_{\alpha} \otimes \boldsymbol{k}_{\beta}\right):\left(\boldsymbol{e}_{3} \otimes \boldsymbol{e}_{k}\right) \delta z \mathrm{~d} x \mathrm{~d} y \\
= & \int S_{\alpha \beta} \boldsymbol{k}_{\alpha} \otimes \boldsymbol{k}_{\beta}: \partial_{k}\left(\boldsymbol{e}_{3} \otimes \boldsymbol{e}_{k} \delta z\right) \mathrm{d} x \mathrm{~d} y \\
= & \int S_{\alpha \beta} \frac{\partial z}{\partial x_{\beta}} \frac{\partial \delta z}{\partial x_{\alpha}} \mathrm{d} x \mathrm{~d} y \\
= & \frac{1}{2} \int S_{\alpha \beta} \delta k_{\alpha \beta} \mathrm{d} x \mathrm{~d} y,
\end{aligned}
$$

where $\delta k_{\alpha \beta}=\boldsymbol{k}_{\alpha} \cdot \delta \boldsymbol{k}_{\beta}+\delta \boldsymbol{k}_{\alpha} \cdot \boldsymbol{k}_{\beta}$.

The same result can be obtained when the horizontal equilibrium is taken into account, and the variation of the shell is now allowed to move in $x$ and $y$ directions. Since the virtual work is a scalar, its expression translated to a curvilinear coordinate system can easily be obtained as

$$
\delta w=\frac{1}{2} \int \bar{S}^{\alpha \beta} \delta h_{\alpha \beta} \mathrm{d} \bar{a},
$$

where $\mathrm{d} \bar{a}$ is an area element projected on to the $x y$ plane, $h_{\alpha \beta}$ is the first fundamental form of the shell, $\bar{S}^{\alpha \beta}$ is the second PK stress tensor having the projected geometry as its reference configuration.

\section{Characteristic lines $=$ asymptotic lines}

A characteristic line of a second-order PDE of the form

$$
A \frac{\partial^{2} z}{\partial x^{2}}+B \frac{\partial^{2} z}{\partial x \partial y}+C \frac{\partial^{2} z}{\partial y^{2}}=-\rho
$$

can be obtained as follows. A characteristic line is a curve whose tangent $(\mathrm{d} x, \mathrm{~d} y)$ is orthogonal to the direction $(\alpha, \beta)$ that is a root of $A \alpha^{2}+B \alpha \beta+C \beta^{2}=0$. Hence, substituting $(\alpha, \beta)=(\mathrm{d} y,-\mathrm{d} x)$, one gets $A \mathrm{~d} y^{2}-B \mathrm{~d} x \mathrm{~d} y+C \mathrm{~d} x^{2}=$ 0 .

In this paper

$$
\frac{\partial^{2} \phi}{\partial x^{2}} \mathrm{~d} x^{2}+\frac{\partial^{2} \phi}{\partial x \partial y} \mathrm{~d} x \mathrm{~d} y+\frac{\partial^{2} \phi}{\partial y^{2}} \mathrm{~d} y^{2}=0,
$$

which is the same as the definition of the asymptotic lines.

\section{Candela's Xotimilco restaurant}

A stress function $\phi(u, v)=B u^{2}-\left(v-\frac{1}{2}\right)^{2}+C v$ and a form diagram $(x, y)=\left(-u, B u^{2}+v+A\right)$ yields a force diagram $(X, Y)=(4 B u v, 2 v+C-1)$. With parameters $A=-\tan (2 \pi / 16)-B, B=(1.06)^{2}$, and $C=15.2, u$ moves from -1 to $1, v=0$ when on the edge, and $v=$ $-A$ when at the center, a shell reproducing the Candela's Xotimilco restaurant can be obtained (Fig. 29).
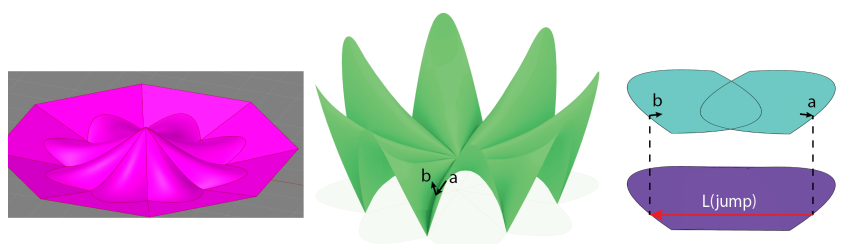

Figure 29. (left to right) stress function, Candlea shell and force diagram. 